\title{
CARCINOMA HEPATOCELULAR E A POSSIBILIDADE DE TRANSPLANTE DE FÍGADO: AVALIAÇÃO PSICOLÓGICA E ASPECTOS EMOCIONAIS
}

\author{
Hepatocellular carcinoma and possibility of liver transplantation: \\ evaluation and emotional aspects
}

\author{
Maria Isabel Warwar, Ilka de Fátima Santana Ferreira Boin
}

\begin{abstract}
RESUMO
Transplante de fígado é a alternativa terapêutica para os pacientes portadores de doenças hepáticas terminais, incluindo o carcinoma hepatocelular (CHC). Após estar inserido na lista, há o alívio para o paciente e, logo após, a constatação da espera, onde eles não têm controle algum. Desse modo, o período de espera é o mais estressante, pois a doença está em atividade plena. Muitas vezes, a piora clínica começa a ficar mais evidente, frequentemente resultando em hospitalizações prolongadas. Durante essa etapa, podem aparecer contraindicações clínicas que inviabilizam o procedimento de transplante, como infecções, danos cerebrais e instabilidades hemodinâmicas. Nesse momento, o psicólogo e a ajuda religiosa podem auxiliar tanto pacientes quanto familiares nessa transição e prepará-los para o eventual período de luto. A experiência mostra-nos que certo nível de ajuste psicológico é de extrema necessidade para o sucesso no pré e pós-transplante em pacientes portadores de CHC. A contribuição do psicólogo é introduzir e respeitar a dimensão subjetiva do paciente durante todo o processo de transplante. Por esse motivo, quanto mais cedo a equipe transplantadora detectar problemas psicossociais, seja de cognição ou de comportamento, pode-se ter a oportunidade de desenvolver planos de tratamento, minimizando qualquer impacto negativo que esses problemas possam causar ao sucesso do transplante.
\end{abstract}

Descritores: Transplante de Fígado; Alcoolismo; Câncer de Fígado; Psicologia Aplicada.

\author{
Instituição: \\ Unidade de Transplante Hepático do Hospital de Clínicas da \\ Unicamp, Campinas/SP \\ Correspondência: \\ Ilka Boin \\ Rua Aldo Oliveira Barbosa 184 - CEP 13086-030, Campinas/SP \\ Fone: (19) 3521-8581 \\ E-mail: ilkaboin@gmail.com
}

Recebido em: 06/09/2013

Aceito em: 30/09/3013

\section{INTRODUÇÃO}

As principais causas da doença hepática são: contaminação pelo vírus da hepatite $\mathrm{B} / \mathrm{C}$, uso abusivo de álcool, hepatite autoimune, cirrose biliar primária ou secundária e esteatohepatite não alcóolica.

Quando o fígado adoece, como consequência, pode surgir a cirrose, que é um processo de várias formas necroinflamatórias, que levam à fibrose hepática, disfunção hepatocelular e remodelamento vascular. Outras complicações decorrentes da doença hepática avançada incluem a ascite, encefalopatia hepática, hipertensão portal com varizes esofagogástricas, disfunção renal e carcinoma hepatocelular (CHC). 
$\mathrm{O}$ CHC é atualmente o quinto câncer mais comum em homens e o oitavo em mulheres. A incidência tem aumentado no ocidente nos últimos anos, em função da obesidade e do uso abusivo do álcool. O principal fator de risco para o surgimento do $\mathrm{CHC}$ é a cirrose hepática.

$\mathrm{O}$ CHC é a sétima causa de óbitos relacionados à neoplasia. Em aproximadamente $90 \%$ dos casos, existe a infecção pelo vírus da hepatite $\mathrm{B}$ e/ou $\mathrm{C}$. $\mathrm{O}$ aumento da incidência de $\mathrm{CHC}$ está diretamente relacionado ao aumento da infecção pelo vírus da hepatite C. ${ }^{1}$

Os sintomas não são específicos, pois dependem da etiologia de base, mas, a partir do momento em que ele se instala no organismo, a evolução da lesão é rápida e constante, dobrando de volume em quatro ou seis meses. Sendo assim, a melhor opção ainda é o transplante de fígado.

Estudos recentes relatam que a taxa de sobrevida, após o procedimento de transplante, encontra-se em torno de $83 \%$ no primeiro ano. ${ }^{2}$ Como causa do sucesso do procedimento, podemos citar a qualidade da técnica cirúrgica, conhecimentos adequados dos métodos de imunussupressão, preservação de órgãos e esquemas eficazes de profilaxia para infecção no pós-operatório.

No Brasil, o Sistema Nacional de Transplantes (SNT), ligado ao Ministério da Saúde, preconizou um sistema de ingresso na lista de transplante de fígado. Para que o paciente portador de $\mathrm{CHC}$ possa ser inserido, ele precisa apresentar comprovação de um tumor de até $5 \mathrm{~cm}$ ou até três tumores de $3 \mathrm{~cm}$ cada, através de exames de imagem. A equipe transplantadora precisa comprovar esses fatos perante uma Comissão Técnico Científica da Secretaria de Saúde do Estado de São Paulo (SP). A partir do momento em que esse paciente é inserido em lista, ele ganha 20 pontos; após três meses de tempo em lista, ele ganha mais quatro pontos, totalizando então 24 pontos; após seis meses em lista, ele ganha mais cinco pontos, totalizando 29 pontos. A alocação de órgãos na lista de transplante funciona por gravidade da doença, sendo, portanto, muito dinâmica. Sem CHC, os pacientes que esperam pelo transplante têm que apresentar uma pontuação mínima de 11 pontos ou de 15 pontos no estado de São Paulo, para entrar na lista de espera. Essa pontuação mínima, para ser inserido em lista, é obtida através do MELD (Model of End-Stage Liver Disease). ${ }^{3,4}$

\section{Avaliação psicológica no período pré-transplante}

O objetivo principal da avaliação psicológica inicial é o de encorajar o doente a ser transplantado, oferecendo subsídios emocionais e cognitivos, para que ele passe pelo procedimento, ${ }^{5}$ pois o objetivo principal do transplante de fígado é o prolongamento do tempo e da qualidade de vida desses pacientes. Outro fator importante, observado na literatura, é o aumento significativo da qualidade de vida desses pacientes após o transplante. ${ }^{6}$
O processo de avaliação consiste em atenuar as preocupações e aceitar o transplante como a única decisão viável para a sua sobrevivência.?

Nesse momento, a orientação ao paciente, utilizando o máximo de informação sobre o tratamento, é de extrema importância, pois, quanto mais informação o ele agrega, menos ansiedade é gerada durante os procedimentos no pré ou pós-transplante. ${ }^{8}$

Na prática, na Unidade de Transplante Hepático do Hospital de Clinicas da Universidade Estadual de Campinas, a avaliação consiste em três momentos pré-definidos e pré-agendados no Serviço de Psicologia, que funciona como livre demanda, tanto da parte dos pacientes, como dos profissionais da área. Portanto, havendo necessidade mútua ou unilateral, haverá acompanhamento psicológico.

$\mathrm{O}$ primeiro momento acontece quando a equipe transplantadora encaminha o paciente para avaliação psicológica, após constatação da necessidade clínica de inserção na lista para transplante de órgãos. Essa avaliação acontece com os pacientes e seus familiares, quando é realizada uma entrevista aberta, contendo dados pessoais, cotidiano, dinâmica familiar, relato de uso de álcool ou outras substâncias, comorbidades psiquiátricas, reação frente à hospitalização prolongada ou não e outros fatores socioeconômicos e culturais.

Após essa avaliação, a equipe multiprofissional reúne-se (semanalmente) e, nesse momento, todos os profissionais dão seu parecer para que seja definido se o paciente tem condições ou não de entrar em lista de transplante de órgãos.

Em um segundo momento, após o paciente ser inserido em lista, há uma reavaliação para situá-lo, em função do que ficou decidido na reunião e dar o suporte necessário perante a decisão tomada pela equipe e durante a espera do procedimento.

Em um terceiro momento, há encontros após o transplante realizado, seja na UTI, enfermaria ou no ambulatório.

É muito importante que o serviço de psicologia observe todo o procedimento de avaliação, pois é de extrema importância que o paciente tenha cognição para que haja adesão a todo o procedimento e tratamento, antes e depois do transplante. Assim, obtém-se a visão de como ele irá se comportar durante o tratamento. ${ }^{9}$

Um trabalho recente mostrou que $12 \%$ dos pacientes que foram inseridos em uma lista de transplante de fígado apresentavam nível de ansiedade moderado e, em $27 \%$, foi relatado como leve. ${ }^{8}$

É de extrema importância observar que a atitude do paciente diante do transplante depende das características subjetivas de cada um. O paciente necessita de habilidade de adaptação, pois haverá alteração nas relações sociais, tendo que construir novas perspectivas, com revisão de suas metas de vida. 
Podemos observar que $71 \%$ desses pacientes apresentaram como etiologia a cirrose alcoólica ${ }^{9,10}$ e, por esse motivo, é necessária avaliação psicológica concreta e dinâmica para poder inserí-lo em lista, pois ele e seus familiares precisam conscientizar-se de que o consumo de álcool não será mais compatível com sua vida, seis meses antes e nunca mais após o transplante.

O tempo é fator importante nessa avaliação, pois, se o CHC aumentar de tamanho, o paciente ficará fora do critério para poder receber um fígado novo, em função das leis vigentes em nosso país. Não sendo submetido ao transplante, outros tratamentos paliativos serão propostos para diminuição do tumor.

Há sentimentos em relação ao transplante, como esperança de um futuro saudável, temor pela cirurgia, possibilidade inerente do transplante falhar, ânsia em prosseguir a avaliação e a preocupação com o tempo, conforme já explicado anteriormente.

A avaliação psicológica precisa conter o histórico do paciente, bem como a dinâmica familiar e profissional à qual está inserido. Há contraindicações psiquiátricas que inviabilizam a entrada desse paciente em lista: contraindicações absolutas sérias (uso abusivo de substâncias ilícitas, sintomas psicóticos, ideação suicida e demência). Há também contraindicações relativas (falta de suporte social do paciente e/ou familiar, desordem de personalidade que limite o relacionamento com a equipe, resultando assim, na não aderência a todo tratamento).

Após estar inserido na lista, há o alívio e, logo após, a constatação da espera, onde os pacientes não têm qualquer controle. Desse modo, o período de espera é o mais estressante, pois a doença está em atividade plena. A piora clínica muitas vezes começa a ficar mais evidente, frequentemente resultando em hospitalizações prolongadas.

Durante essa etapa, podem aparecer contraindicações clínicas que não viabilizam mais o procedimento de transplante, como infecções, danos cerebrais e instabilidades hemodinâmicas. Nesse momento, o psicólogo e a ajuda religiosa podem auxiliar os pacientes e seus familiares, preparando-os para eventual período de luto.

A experiência mostra-nos que certo nível de ajuste psicológico é de extrema necessidade para o sucesso no pré e pós-transplante em pacientes portadores de CHC. A contribuição do psicólogo é introduzir e respeitar a dimensão subjetiva do paciente durante todo o processo de transplante. Por esse motivo, quanto mais cedo a equipe transplantadora detectar problemas psicossociais, seja de cognição ou de comportamento, poderá ter a oportunidade de desenvolver planos de tratamento, minimizando qualquer impacto negativo que esses problemas venham causar ao sucesso no transplante.

\section{A avaliação psicológica no pós-transplante}

Observamos que a sobrevida no primeiro ano póstransplante varia de $70-80 \%$ e é de $50 \%$ no final do quinto ano, de acordo com dados da Associação Brasileira de Transplantes de Órgãos (ABTO) e dados do Sistema Estadual de Transplantes da Secretaria de Estado da Saúde do Estado de São Paulo. ${ }^{4}$

Observamos que, em média, $40 \%$ dos pacientes apresentam ansiedade ou depressão antes do procedimento de transplante. Após o procedimento, $30 \%$ continuam apresentando ansiedade e/ou depressão durante o primeiro ano pós-transplante. Isso pode ser explicado pelo fato que, após um ano, a sobrevida diminui, o que os deixa excessivamente preocupados com o futuro. A ansiedade é explicada pela preocupação excessiva com o funcionamento do enxerto, complicações clínicas e efeitos colaterais das medicações. O que se observa na prática clinica é que pacientes que esperam pelo procedimento em condições clínicas piores, se comparados com outros, têm maior incidência dessas patologias mentais após o procedimento. ${ }^{9}$

Observamos, também, que $44 \%$ dos pacientes têm grande chance de apresentar algum sintoma de estresse póstraumático, pois, de maneira recorrente, aparecem visões do que se passou, pesadelos, delírios e alucinações durante os primeiros anos pós-transplante. ${ }^{9-11}$

Existem intervenções predefinidas para que o paciente apresente comportamento adequado e esperado pela equipe transplantadora, que podem ser educativas, comportamentais e psicológicas. A intervenção educativa acontece no momento do pré-transplante, onde há informação do procedimento através de palestras ou recursos audiovisuais informativos. As intervenções comportamentais são utilizadas no uso de reforços, punições e constatação de mudanças positivas em outros pacientes. As intervenções psicológicas acontecem através de suporte emocional (psicoterapia breve e/ou aconselhamentos), suporte social e intervenções com ajuda da equipe multiprofissional. ${ }^{12,13}$

Há níveis de atuação dessas intervenções:

01. Somente com o paciente

02. Com o cuidador do paciente

03. Com os centros de saúde e pastorais

04. Com o sistema de saúde municipal

Encontramos, com facilidade, algumas perturbações psiquiátricas no pós-transplante, como depressão, ansiedade, delirium (como efeitos secundários à medicação) e recidiva de alcoolismo. ${ }^{14,15}$

Outro fator precisa ser levado em consideração, no período pós-transplante, é a qualidade de vida, que, por definição, está diretamente relacionada ao impacto do estado de saúde sobre o bem estar de cada paciente. Podemos afirmar que o 
transplante de fígado, na maioria das vezes, traz ao doente melhora na sua qualidade de vida. ${ }^{14-16}$

Em nosso serviço, a psicologia atua em diversas frentes para que a qualidade de vida seja alcançada, como, por exemplo, no pós-operatório imediato, atuando nas visitas aos leitos da UTI/Enfermaria e dando suporte emocional aos familiares durante todo o processo. É importante salientar que a estadia na UTI pode ser longa, bem maior que o esperado, havendo vários procedimentos invasivos, que acabam prejudicando emocionalmente o paciente e seus familiares, durante todo o processo. Cabe ao psicólogo, nesse momento, fazer uma "ponte" de orientação entre o paciente, seus familiares e equipe médica, priorizando sempre a informação coerente em todas as etapas, para reduzir as sequelas emocionais, por conta da ansiedade dessa situação.

Após a alta hospitalar, esses pacientes têm consultas préagendadas, onde é preenchido outro protocolo de avaliação no período pós-transplante, para que sejam obtidas informações sobre a vivência do transplante.

\section{Uso abusivo do álcool}

A ingestão abusiva de álcool ainda é uma das principais causas para o transplante de fígado. Devido a fatores sociais e morais, os indivíduos com doença hepática alcoólica nem sempre têm condições de competir de forma igualitária para a submissão ao transplante. ${ }^{11}$

O diagnóstico de doença hepática alcoólica (DHA) não é simples, e o único exame para diagnóstico médico é a biópsia hepática, que acarreta um risco significativo ao doente com cirrose hepática.

Vários autores relatam que não há concordância entre a quantidade de álcool consumida, diagnóstico psiquiátrico de abuso ou dependência e o desenvolvimento de DHA.

A entrevista psicológica inicial, bem como a avaliação da dinâmica familiar, são de extrema importância e, durante este contato, devemos considerar:

01 - Tempo de abstinência, pré-definida como seis meses, para o paciente poder ser inserido em lista.

02 - Suporte social, determinante para a recaída ou não no pós-transplante.

Quando houver recaída durante o processo no prétransplante e esse paciente encontrar-se ativo na lista de espera, ele precisará ser retirado de lista, reavaliado novamente em relação ao uso abusivo de álcool, acolhido em tratamento para chegar à abstinência novamente, e passar por nova espera de seis meses para poder ser reinserido em lista.

\section{Considerações finais}

O que devemos ter em mente é que, na maioria dos casos, o transplante de fígado é a única possibilidade de sobrevida em pacientes com $\mathrm{CHC}$, e essa possibilidade não oferece, aos mesmos, um tempo confortável para a espera em lista.9

Podemos afirmar, com toda convicção, que esses pacientes, após o transplante de fígado, apresentam qualidade de vida renovada, com suas atividades cotidianas restauradas, na maioria dos casos.

O importante é que a ajuda psicológica, bem como uma equipe multiprofissional coesa e dinamicamente atuante, são os diferenciais para que esses pacientes e seus familiares possam passar pelo procedimento de transplante da melhor maneira possível.

\section{ABSTRACT}

Liver transplantation is a therapeutic alternative for patients with end-stage liver diseases including hepatocellular carcinoma. After being inserted on the waiting list, there is relief, and shortly, the perception of the waiting, where patients have no control. Thus, the waiting period is the most stressful, as the disease is fully active. Often, clinical deterioration becomes more apparent, resulting in prolonged hospitalization. During this step, it may appear clinical contraindication which may make the transplant impossible, such as infections, brain damage and hemodynamic instabilities. At this occasion, the psychologist and religious assistance can help both patients and families in such transition and to prepare them for a mourning period.

Experience shows that a certain level of psychological adjustment is required for a successful pre- and post-transplant in patients with HCC. Contribution of a psychologist is to introduce and respect the patient's subjective dimension throughout the transplant process. For this reason, the earlier the transplant team detects cognition or behavior psychosocial issues, it may have opportunity to develop treatment plans to minimize any negative impact these problems can cause to a successful transplantation.

Keywords: Liver Transplantation; Alcoholism; Liver Neoplasms; Psychology, Applied. 


\section{REFERÊNCIAS}

1. Fernandes JH, Millan LS, Massarollo PCB, Mies S. Efeito da escala MELD na mortalidade após transplante de fígado - Rev Med (SP). 2002;81(1/4):15-21.

2. Freitas ACT, Parolin M, Stadnik L, Coelho J. Carcinoma hepatocellular: impacto do tempo em lista e das formas de tratamento pré-operatório na sobrevida do transplante de fígado cadavérico na era pré-MELD em um centro no Brasil. Arq Gastroenterol. 2007;44(3):189-94.

3. www.saude.sp.gov.br/transplante - Sistema Estadual de Transplantes - acesso em novembro de 2013.

4. www.snt.gov.br - cadastro técnico de fígado - acesso em novembro de 2013. Lucey MR.

5. Liver transplantation for alcoholic liver disease.Clin Liver Dis. 2007 May;11(2):283-9.

6. Totti V, Zancanaro M, Trerotola M. Costa A. Quality of life and energy expenditure in transplant recipient football players. Transplant Proc. 2013; 45:2758-60.

7. Sendas S, Perdigot R, Abrunheiro L. Avaliação e acompanhamento psicológico pré e pós transplante hepático. Psicologia, Saude \& Doenças. 2005;(6):139-43.

8. Santos GR, Boin IF, Pereira MI, Bonato TC, Silva RC, Stucchi RS et al. Anxiety levels observed in candidates for liver transplantation. Transplant Proc. 2010;42(2):513-6.
9. Errichiello L, Picozzi D, Notaris E. Prevalence of psychiatric disorders and suicidal ideation in liver transplanted patients: A cross-sectional study. Clin Res Hepatol Gastroenterol, 2013.

10. Weinrieb RM1, Van Horn DH, McLellan AT, Lucey R. Interpreting the significance of drinking by alcohol-dependent liver transplant patients: fostering candor is the key to recovery. Liver Transpl. 2000;(6):769-76.

11. Warwar MI, Boin IFS. Expectativa da recidiva de álcool em pacientes portadores de cirrose hepática. J Bras Transplant. 2012;15(3):1656-9.

12. Telles Correia, D. Candidatos a transplante hepático com doença hepática alcoólica. Acta Med Port. 2011;(24): 835-44.

13. Miller LR, Paulson D, Eshelman A, Bugenski M, Brown KA, Moonka D, et al. Mental health affects the quality of life and recovery after liver transplantation. Liver Transpl. 2013;19(11):1272-8.

14. Telles Correia D, Barbosa A, Barroso E, Monteiro E. Abordagem psiquiátrica do transplante hepático. Acta Med Port 2006;(19):16580.

15. Bleser L, Matteson M, Dobbels F, Russell C, De Geest S. Interventions to improve medication-adherence after transplantation: a systematic review. Transplant Int. 2009;(2):780-97.

16. Lazzareti CT. Transplantre de orgãos: avaliação psicológica. Psicol. Argum, 2006;24(45):35-43. 